\title{
EL MEJOR DINERO POSIBLE: UN COMENTARIO
}

\author{
PHILIPP BAGUS*
}

\section{I \\ INTRODUCCIÓN}

Sánchez Martínez, L.C. (2009) acierta en varios argumentos de su artículo sobre el mejor dinero posible. Estamos de acuerdo que el dinero debería ser el resultado del acuerdo voluntario de los individuos, no debería ser impuesto por los gobiernos y que los ciudadanos deberían poder elegir la moneda más eficaz.

$\mathrm{El}$ autor entiende por dinero fiduciario un dinero «sin respaldo en bien tangible alguno» (p. 173). No estamos de acuerdo, en primer lugar, cuando se refiere a la aparición del dinero, en concreto el hecho de que el dinero fiduciario podría surgir sin la ayuda del estado porque sería más eficiente. En segundo lugar, tampoco estamos de acuerdo con que el «dinero fiduciario pueda existir sin ningún tipo de respaldo estatal y ser el mejor dinero posible.» (p. 174).

Para justificar nuestro desacuerdo vamos primero a analizar como surge el dinero en el mercado libre. Después discutiremos cómo surgió el dinero fiduciario, cómo compite con el dinero mercancía y discutiremos los ejemplos que el autor considera como pruebas de la aparición de un dinero fiduciario. Finalmente hablaremos sobre la banca con reserva fraccionario que para Sánchez Martínez no implica ningún problema económico ni jurídico.

* Doctor en Economía y Profesor de Economía de la Universidad Rey Juan Carlos. 


\section{II EL SURGIMIENTO DEL DINERO}

En una economía de trueque existe el problema de la doble coincidencia de necesidades. El señor A tiene que valorar más lo que tiene el señor B y de lo que él carece y viceversa. Supongamos que A quiere un queso y tiene zapatos. Encuentra al señor B que tiene un queso pero lo que quiere son huevos. No habrá intercambio directo. Así las posibilidades de intercambio en una economía de trueque son muy reducidas, lo que restringe la división del trabajo y así la productividad y el nivel de vida. Los medios de intercambio aparecen en una economía de trueque cuando unos individuos se dan cuenta de que unas mercancías son más frecuentemente intercambiadas que otras. Se venden más fácilmente. Ciertos empresarios demandan estas mercancías, no para usarlas como bien de consumo o factor de producción, sino para intercambiarlas a cambio de lo que necesitan de veras; es decir, las usan como medio de intercambio. Supongamos que A se da cuenta de que unas piezas de metal (plata) se intercambian más frecuentemente que otras. De esta manera cuando A vende su producto, en nuestro caso zapatos, no demanda lo que necesita directamente, queso, sino que demanda lo que se intercambia más frecuentemente: piezas de plata. Compra plata y la usa como medio de intercambio para comprar lo que necesita. El medio de intercambio tiene entonces dos demandas solapadas. Por una parte para usarlo como bien de consumo o factor de producción (joyería) y por otra como medio de intercambio. En la medida en que el empresario tiene éxito con su estrategia del cambio indirecto, se refuerza el proceso. El señor A puede repetir su acción y otros individuos le imitan. Entonces cada vez más personas usan el medio de intercambio hasta que uno de ellos se convierte en el medio de intercambio común y generalmente aceptado. Surge así el dinero en un proceso cumulativo (Menger, 1871, 1892).

En este proceso competitivo de la aparición del dinero suelen prevalecer unas mercancías con unas características específicas. De las encontradas por Carl Menger, Sánchez Martínez menciona las siguientes: escasez, buena distribución geográfica, divisibilidad, bajo coste de transporte y atesoramiento y durabilidad ilimitada. 
Admite Sánchez Martínez que el oro y la plata cumplían perfectamente estos requisitos pero añade que el dinero fiduciario emitido en billetes y monedas los cumplirían aún mejor. Este argumento podría implicar que esta es la razón por la que aparece el dinero fiduciario, porque cumple mejor estas exigencias. Encontramos sin embargo varios problemas con esta tesis.

En primer lugar, el autor se confunde con el antes y el después. Menger (1892) menciona las características que una mercancía tuvo que tener antes de convertirse en dinero, es decir, las que debería tener una mercancía antes de tener una amplia demanda solapada como medio de intercambio, no después de que haya surgido como dinero.

Solo una vez que el dinero sea fiduciario a través de procesos, que comentaremos en breve, puede cumplir estos requisitos. Pero ¿puede un dinero fiduciario como el papel moneda cumplirlos antes de que sea ya dinero?, ¿puede prevalecer el papel sobre otras mercancías como el oro en el proceso competitivo a través del cual surge el dinero?

El papel no puede competir con una mercancía como el oro por varias razones. El papel como mercancía tiene un coste de atesoramiento más alto que el oro. Hay que almacenar una cantidad enorme de papel en comparación con el oro porque éste tiene mucho más valor que aquél. El equivalente de un coche en papel, son toneladas de papel, cuyo coste de almacenamiento es altísimo en comparación con el coste de almacenamiento de unas pocas onzas de oro que compran un coche.

Luego los costes de transporte del papel son también mucho más altos. Es más barato transportar unas onzas de oro que unas toneladas de papel. Además el papel era y es menos escaso que el oro. El oro tenía mucho más valor por peso en una economía de trueque que el papel. Es cierto, que una vez que tengamos dinero fiduciario, el papel moneda puede tener mucho valor. Pero se trata de explicar como podría llegar a eso en un proceso de mercado.

En segundo lugar, Sánchez Martínez no menciona otras características que incrementan la negociabilidad. En relación con esto Menger comenta que la resistencia de una mercancía la hace más negociable y útil como medio de intercambio. El oro es más resistente y durable que el papel. Más importante aún es que para la 
mercancía existan muchos deseos insatisfechos y que la intensidad de los deseos sea alta y permanente (Menger 1892). Esta demanda constante de la mercancía incrementa su negociabilidad, algo esencial para la aparición del dinero. Hace que la mercancía sea un gran depósito de valor, funciona como un «seguro» que mantiene el valor del bien incluso cuando de desmonetiza (Bagus 2009, 50). El oro tiene una gran demanda industrial y de consumo lo que mantiene su valor aunque se desmonetice, mientras la diferencia entre el valor monetario y el valor desmonetizado del papel moneda es mucho mayor.

Ya hemos visto que el dinero surge en un proceso competitivo en el que una demanda monetaria se solapa con la demanda industrial de una mercancía muy negociable. No puede ser de otra manera. El origen siempre está en una mercancía (Rothbard $2008,15)$. Nadie puede declarar que un material sin valor se convierta en dinero, ni una comunidad puede decidir que algo sin valor se convierta en dinero. Esto es imposible porque no hay conocimiento del valor que pueda servir de base de la demanda monetaria. La demanda de dinero se basa en el conocimiento del poder adquisitivo del dinero en el pasado ${ }^{1}$ ya que necesita unos precios del pasado. Más concretamente la demanda de dinero de hoy es función de las expectativas del precio del dinero el día de mañana que, a su vez, están basadas en el precio del dinero ayer. $^{2}$ Y la demanda de dinero de ayer era función de las expectativas del precio del dinero de hoy, que a su vez están basadas en la experiencia del precio del dinero de anteayer. Y así sucesivamente hacia atrás en el tiempo hasta el día en que una determinada mercancía empieza a tener una demanda solapada como medio de intercambio. Es decir, el proceso siempre empieza en una mercancía con una demanda como bien de consumo o factor de producción a la que se añade la demanda monetaria.

\footnotetext{
1 Ese es el teorema regresivo del dinero de Ludwig von Mises (1912).

2 Sánchez Martínez considera que todo el dinero tiene una demanda fiduciaria; también el oro porque «se basaba en la fe de que el oro iba a ser aceptado para el intercambio comercial.» La verdad es que la demanda monetaria del oro se basaba en las expectativas de su poder adquisitivo en el futuro. De hecho cualquier factor de producción es comprado por los empresarios en función de sus expectativas del precio que va a tener el producto de consumo a que contribuye en el futuro.
} 
Nadie puede crear dinero a partir de un material sin valor. Si yo emito un dinero fiduciario nuevo llamado «Bagus» no existe un conocimiento de precios pasados. Si intento comprar un coche con un billete que pone «100 Bagus» lo más probable es que no pueda comprar el coche. El vendedor no tiene experiencia de precios del pasado. No sabe lo que compraban «100 Bagus» ayer. Puedo intentar engañarle y decirle que «100 Bagus» ayer compraban un coche o un kilo de oro y seguro que mañana también lo harán. Pero no hay altas probabilidades de convencer al vendedor ni mucho menos al resto de la población. Sin embargo Sánchez Martínez piensa que se puede introducir un dinero fiduciario sin respaldo en bien tangible alguno basándose única y exclusivamente en la confianza (184). ${ }^{3}$ Lo contrario es cierto, el dinero siempre surge en un proceso de mercado a partir de la demanda de una mercancía que se intercambia con más facilidad.

El dinero libremente elegido siempre es una mercancía. Otra cuestión es que el Estado pueda introducir el dinero fiduciario con su monopolio de violencia corrompiendo el dinero mercancía. Puede suspender el pago en metálico de certificados de depósito y declararlos como dinero de curso legal. Este cambio en la historia no fue radical pero tampoco provisional. Fue en el 1971 cuando desapareció el último vínculo con el oro.

De esta manera se tiene un conocimiento de los precios del dinero del pasado. El día de la suspensión del pago en metálico se conocen los precios del día anterior y éstos pueden servir de base para las expectativas y demanda del dinero fiduciario. Esta violación de la ley, la suspensión del pago en metálico, y la introducción de una ley de curso forzoso sólo lo puede efectuar el Estado. Así se resuelve el problema del conocimiento de precios pasados. En cambio los empresarios privados sólo podrían introducir dinero fiduciario si la gente lo vinculara con un dinero

3 Añade que el dinero fiduciario tendría la ventaja de una producción barata. Si se usa oro como dinero hay que abrir una mina que es mucho más costoso que imprimir dinero o introducir unos apuntes contables en un ordenador. El misterio que Sánchez Martínez no explica es cómo algo que antes no tenía valor (un billete de papel o un apunte informatico) de repente puede llegar a tenerlo. Además es una ventaja del oro que su producción sea muy cara porque da seguridad ya que su cantidad no se puede expandir facilmente. 
mercancía surgido antes. Si digo que «100 Bagus» valen por 1 kilo de oro, siendo el oro un medio de intercambio generalmente aceptado, se pueden calcular los precios en términos de «Bagus» de ayer. Pero es muy dudoso que alguien me lo vaya a aceptar por lo que le digo, aunque asegure que no voy a incrementar la masa monetaria. Estaría encantado si el autor aceptara mi billete por 1 kilo de oro.

III

\section{DINERO FIDUCIARIO EN LA COMPETENCIA}

Ya vimos que un medio de intercambio generalmente aceptado de carácter fiduciario sólo puede surgir por una violación contractual y con el apoyo del Estado. Sánchez Martínez mantiene también que existiendo el dinero fiduciario puede sobrevivir en competencia con el dinero mercancía. Pero ¿podrían mantenerse el euro o el dólar si privatizamos su emisión y abolimos los leyes de curso forzoso?

El gran inconveniente de estas monedas es que su valor se basa práctica y exclusivamente en la demanda monetaria. La demanda no monetaria prácticamente no existe, lo que supone una ventaja competitiva importante. Cuando se desmonetizan pierden prácticamente todo su valor, lo que incrementa el riesgo de mantenerlas porque al día siguiente pueden no valer nada. Sólo dependen de la confianza.

Si se desmonetizara otro medio de intercambio, como el oro, sólo perdería una parte de su valor porque la demanda no monetaria lo soportaría. La demanda no monetaria es como un "seguro» contra la desmonetización. Además le confiere estabilidad. Cambios en la demanda monetaria son matizados por la continua demanda industrial. El dinero mercancía suele fluctuar menos en valor que el valor de un dinero fiduciario que sólo se basa en la confianza que, evidentemente, puede fluctuar mucho. De esta manera a largo plazo es probable que gane el oro en la competencia con el dinero fiduciario.

Además es posible que el dinero fiduciario se desmonetice inmediatamente si el estado deja de soportarlo. Sánchez Martínez 
argumenta que eso no pasaría y da tres argumentos. Escribe que las leyes de curso forzoso no son necesarias para que circule el dinero fiduciario dado que dólares y euros circulan en países dónde no tienen esta consideración. Es verdad que los dólares circulan en países dónde no son de curso forzoso, pero eso no significa que sigan circulando sin el privilegio de ser moneda legal en los EE.UU. La demanda de dólares fuera de los EE.UU. se basa en el hecho de que sean dinero de curso forzoso en este país.

También escribe que las reservas o activos de los bancos centrales no hacen circular el dinero fiduciario. Esto implica que el dinero fiduciario no necesita las reservas de los bancos centrales para circular. Sin embargo, éstas son importantes para el valor de una moneda, dado que aumentan la confianza en ella y pueden ser usadas para defender su valor comprando la moneda, rescatar el sistema bancario, reformar el sistema etc. (Bagus y Schiml 2009). Sin el respaldo de las reservas de la Reserva Federal el dólar podría perder mucho de su valor e incluso desaparecer.

Tampoco cree Sánchez Martínez que el apoyo oficial del gobierno juegue un papel importante en el valor de una moneda. Sin embargo un gobierno puede subvencionar su moneda con impuestos. Por ejemplo, puede entregar activos al banco central o al sistema bancario y así reforzar la confianza en ellos (Bagus 2009). Durante la Guerra Civil Americana los monedas de los Estados del Norte y del Sur fluctuaban en función de sus victorias y derrotas como señala Carver $(1934,203)$. Hazlitt $(1978,76)$ comenta el caso de un «peso» emitido por el gobierno japonés durante la segunda guerra mundial en Filipinas. En cuanto el gobierno japonés, que respaldaba este dinero fiduciario, fue expulsado por el ejercito americano el «peso japonés» perdió todo su valor:

One of the most striking illustrations of the importance of the quality of the currency occurred in the Philippines late in World War II. The forces under General Douglas MacArthur had effected a landing at Leyte in the last week of October 1944. From then on, they achieved an almost uninterrupted series of successes. Wild spending broke out in the capital of Manila. In November and December 1944, prices in Manila rose to dizzy heights. Why? There was no increase in the money stock. But the inhabitants knew that 
as soon as the American forces were completely successful their Japanese-issued pesos would be worthless. So they hastened to get rid of them for whatever real goods they could get.

En resumen, el prestigio del gobierno, su capacidad de imponer impuestos, sus activos y las leyes de curso forzoso, aceptando para pagar impuestos sólo la moneda legal, soportan las monedas fiduciarias. Sin este soporte perderían en la competencia con el dinero mercancía.

IV ¿ES TODO EL DINERO FIDUCIARIO?

Sánchez Martínez mantiene que todo dinero sea fiduciario porque su valor depende de la fe con que se aceptará como dinero en el futuro. Aquí parece que existe una confusión semántica. La demanda monetaria de un bien depende de la expectativa de su poder adquisitivo en el futuro. El autor parece querer sustituir el uso de la palabra «expectativa», muy importante en economía por el uso de la palabra «fe». Se oscurece la diferencia esencial entre el dinero fiduciario y el dinero mercancía. El valor del dinero fiduciario, casi exclusivamente, se basa en la confianza de su aceptación en el futuro y el dinero mercancía se basa en una demanda monetaria y una demanda industrial. El oro tenía una gran demanda industrial antes de que se convirtiera en oro. El dinero fiduciario no tiene ninguna demanda industrial antes de que se impusiera a la fuerza como dinero.

\section{$\mathrm{V}$ \\ DOS SUPUESTOS EJEMPLOS DE DINERO FIDUCIARIO PRIVADO}

Sánchez Martínez da dos ejemplos para demostrar que un dinero fiduciario sin respaldo del Estado puede sobrevivir en competencia con dinero mercancía. El primer ejemplo es el Dolár Linden. El Dolár Línden es una moneda que se usa en un juego de internet 
(Second Life). Los creadores del programa vendieron 300 Dólares Linden por 1 US dólar. Así había un conocimiento de los precios del pasado igual a cuando se sustituyó la Peseta por el Euro. Luego se dejó fluctuar el tipo de cambio y los creadores siguieron vendiendo Dólares Linden aumentando la masa monetaria en el juego, concediendo el derecho a reconvertirlos en dólares reales, lo que soportaba su demanda.

La existencia del Linden Dólar no demuestra que pueda sobrevivir un dinero fiduciario en la competencia del mundo real y convertirse en un medio de intercambio comúnmente aceptado. También el juego «Monopoly» tiene su propia moneda y los jugadores la aceptan para pagos en el juego. También los chips de los Casinos se usan como medio de intercambio en ellos. El Linden Dólar se puede usar para pagar, por ejemplo, en una cuenta premium en «Second Life».Su tipo de cambio fluctúa dentro de este juego. Existe una expectativa de canjear el Linden Dólar por la moneda de curso legal dentro de un margen de intercambio. Así el Linden Dólar es soportado por el US dólar. La existencia de la moneda de juego no muestra que podría sobrevivir en competencia con dinero mercancía en la vida real. Una moneda de juego no es un medio de intercambio común y generalmente aceptado.

El otro ejemplo de Sánchez Martínez es el Swiss Dinhar. Después de la Primera Guerra del Golfo el gobierno de Sadam Hussein no pudo importar los Dinhars antiguos producidos con tecnología suiza por el bloqueo económico. Empezó a imprimir nuevos Dinhars, los Sadam Dinhars. Mandó canjear los antiguos Dinhars por los nuevos. Sin embargo, en la región norte de los Kurdos la gente no le hizo caso. Sánchez Martínez cree que eso muestra que un dinero fiduciario (Swiss Dinhars) puede sobrevivir sin el apoyo de un gobierno. Sin embargo, el uso del Swiss Dinhars en el norte de Iraq se puede explicar perfectamente por el apoyo estatal esperado. Como señala Hal Varian:

In fall 2002, as it became more and more likely that the United States would invade, the Swiss dinar became more and more valuable. This appreciation was driven by expectations. If the Kurds had expected that they would once again fall under 
Saddam's sway, the Swiss dinar would have quickly become worthless. As this became less likely, and the belief that future governments would accept the Swiss dinar became more widespread, the local currency became more valuable. Of course, every exchange rate movement can be interpreted in two ways: in the north, the Kurdish regional government initially interpreted the rise in the Swiss dinar against the dollar as a fall in the value of the dollar. The government soon realized, however, that since the dollar was stable against other currencies, the correct explanation was that recounted above: the increasing belief that the Swiss dinars would, in fact, be honored by future governments. The government was right. On July 7, 2003, the American occupation administrator, L. Paul Bremer III, announced the creation of a new Iraqi dinar that would be exchanged for the two existing currencies at a rate that implied that one Swiss dinar would be worth 150 Saddam dinars. (2004)

Las expectativas de un respaldo estatal de la moneda soportaba el valor de los Dinhars. Al final el autor menciona unas monedas locales como prueba de la posibilidad de un dinero fiduciario sin respaldo estatal. Es el caso de la localidad austriaca de Wörgl que emitía «Schwundgeld» en los años 30 del siglo pasado. Aquí se equivoca el autor porque el «Schwundgeld» era respaldado por la moneda de curso legal, el Schilling (Taghizadegan 2008, 40).

La existencia de unas monedas locales y comunidades de trueque se explica por tres razones (Taghizadegan 2008). Primero, muchas comunidades de trueque sirven para evadir impuestos. Es una forma de trueque la ayuda entre vecinos que realizan intercambios sin tener que pagar impuestos. Dentro de comunidades pequeñas estos sistemas de trueque tienen éxito porque disminuyen la carga fiscal. No convierte estos medios en medios de intercambio comúnmente aceptados. Segundo, como consecuencia de la existencia de muchas monedas locales su carácter es estrictamente regional. La gente usa este medio de intercambio para identificarse con su región y conoce personalmente a gran parte de los usuarios de la moneda. Tercero, se impiden los efectos nefastos de la inflación de la moneda estatal. Las monedas 
locales actuales son medios de intercambio complementarios que existen al lado del dinero generalmente aceptado y su valor tiene una referencia con el dinero de curso forzoso.

VI

\section{LA BANCA CON RESERVA FRACCIONARIA}

Sánchez Martínez mantiene que la banca con reserva fraccionaria sería factible porque los individuos podrían pactar lo que quisieran. Cree que toda entrega de dinero a un banco es un préstamo hasta que el cliente pide el dinero (190). Como señala Huerta de Soto (1998) existen grandes diferencia entre un préstamo y un depósito. En los préstamos existen necesariamente plazos. En los depósitos no hay plazos dado que hay una disponibilidad completa y continua a favor del depositante. El elemento esencial de los préstamos es el traslado de la disponibilidad de bienes presentes a favor del prestatario. El elemento esencial de los depósitos es la guarda o custodia del tantundem. Por lo tanto, la obligación en un contrato de depósito es mantener siempre el tantundem a disposición del depositante. La obligación en un contrato de préstamo es devolver el tantundem transcurrido el plazo con los intereses.

En el sistema actual los depositantes entregan su dinero como un depósito y el banco lo usa como un préstamo. Hay un error in negotio. En una sociedad libre cada uno puede ofrecer los bienes y servicios que quiera, pero eso no significa que todos los contratos voluntariamente pactados sean válidos. Puede haber error in negotio, las causas del contrato pueden ser incompatibles o el cumplimiento del contrato puede ser imposible. Por lo tanto Sánchez Martínez ignora los argumentos jurídicos en contra del depósito bancario con reserva fraccionaria.

Luego plantea la siguiente posibilidad:

Si los bancos incrementan los créditos que conceden a cargo de disminuir sus reservas lo que estarán haciendo es provocando una reducción del valor de sus depósitos y por tanto un incremento del precio de los bienes que se pueden adquirir con esos depósitos. 
Lo que reduce el poder adquisitivo de los poseedores de depósitos bancarios pero no el de los poseedores de dinero efectivo (192).

Este autor piensa que los depósitos tendrían un descuento en relación con el dinero en efectivo. Es cierto que instrumentos financieros y medios de pago como pagarés, acciones, préstamos o bonos varían en su precio conforme a la solvencia del deudor. Pero esto no es cierto para los depósitos. La esencia del dinero como institución es que reduce la incertidumbre. Con un saldo de tesorería la gente se puede enfrentar mejor a la incertidumbre del futuro. En un depósito el elemento esencial es la guarda y custodia, causa o motivo del contrato, lo que implica la disponibilidad completa. Incluso el depositante incrementa la disponibilidad cuando cree que el dinero está más seguro en el banco que en su bolsillo. Con la disponibilidad completa y continua no hay descuento en relación con el dinero en efectivo. Los depositantes creen que sus depósitos están completamente disponibles, lo que se manifestó por ejemplo en la indignación de los depositantes en Argentina cuando los bancos no se los devolvieron. Como los depositantes, conforme a la causa del contrato, creen su dinero disponible no existen descuentos de depósitos frente al pago en efectivo hasta el momento en que como en Argentina se suspenden los pagos o se pone en evidencia que un impago pueda ocurrir. De esta manera la expansión de créditos es inflacionista en la medida en que los depositantes consideren sus depósitos a su disposición.

\section{VII UNA ANALOGÍA INVÁLIDA}

Termina Martínez Sánchez con la misma confusión que su artículo ha mostrado antes escribiendo: «Pero defender a las monedas con respaldo de materias primas o metales preciosos como alternativa a las monedas estatales fiduciarias es como defender la desnacionalización del ferrocarril postulando la vuelta de las diligencias de caballos.» Muchas veces las analogías fallan porque no se 
parece un ejemplo al otro en un aspecto clave. Desafortunadamente las analogias pueden causar gran confusión a primera vista. Analicemos la analogia en detalle. Primero, es cierto que se puede pedir la desnacionalización de la moneda y del oro nacionalizado. Luego el mercado podría elegir la moneda que sea más adecuado y hemos analizado las razones por las que a largo plazo los metales preciosos probablemente ganarían a sus competidores y hemos discutido también los problemas de introducir dinero fiduciario en un mercado libre. No se pide volver a un patrón oro intervenido como existía antes sino la completa provatización. Defender la abolición del dinero publico y volver a un dinero privado que por las razones expuestas no puede ser fiduciario es más parecido a defender la abolición de la esclavitud y la vuelta a un mercado libre de trabajo.

Segundo, la frase del autor parece implicar que todo lo que ha sucedido después es automaticamente mejor. El ferrocarril es mejor que los caballos que son un medio de transporte anticuado e ineficiente. Aunque en las tecnólogias parece que siempre hay avance en muchas areas de la sociedad no es así. No todo lo que ha sucedido después es mejor. En la época de los caballos, los impuestos eran más bajos que hoy que tenemos unos ferrocarriles públicos. Sería mejor volver atrás a impuestos más bajos. Sería también mejor volver atrás a un mundo más libre con menos regulaciones y menos estado y menos contaminado en algunas áreas. Igualmente, sería mejor volver a un dinero más privado como un patrón oro. Es decir, no todo lo del pasado es inferior a lo que tenemos hoy. Y no todo lo del futuro será mejor a lo que tenemos hoy. Probablemente tendremos avances técnológicos, pero también es posible que haya menos libertad. Además, en unos 100 años la mayoría de nosotros habremos muerto. ¿Es esto mejor porque es más tarde? Desde el punto de vista individual no es bueno introducir esta faceta del futuro ya hoy. En suma, la analogia de Martínez Sánchez con referencias al mundo técnológico no funciona con el dinero. Un patrón oro no es un regreso sino un gigantesco paso adelante hacía un sistema monetario más estable, justo y libre. 


\section{VIII \\ CONCLUSIÓN}

Acordamos con Martínez Sánchez que el Estado no debería intervenir en temas monetarios y que en una sociedad libre se pueda ofrecer lo que se quiera. La tesis general de Martínez Sánchez no es cierta. El mejor dinero posible no es el dinero fiduciario. El dinero fiduciario no puede surgir en un mercado libre porque no hay conocimiento de precios anteriores para basar la demanda monetaria. La existencia del dinero fiduciario se debe a las intervenciones del Estado que nacionalizó la moneda. Además, el dinero fiduciario no puede sobrevivir en competencia libre con el dinero mercancía porque ese activo sin el apoyo del Estado es muy arriesgado ya que no tiene el seguro de una amplia demanda industrial. Los ejemplos históricos de Martínez Sánchez sólo demuestran la posibilidad de medios de intercambio en ámbitos restringidos como comunidades de trueque o juegos, manteniendo en todo caso sus vínculos con un medio de intercambio generalmente aceptado y la importancia de las expectativas acerca de las intervenciones estatales. En relación con la reserva fraccionaria hay que subrayar que no todos los contratos voluntarios tienen que ser válidos en una sociedad libre por incompatibilidades jurídicas, error in negotio, causas incompatibles, cumplimiento imposible etc. Existe una clara separación jurídica y económica entre un depósito y un préstamo. El elemento esencial del préstamo es el traslado de la disponibilidad de los bienes presentes a favor del prestatario. La causa principal del depósito es la guarda y custodia y así se mantiene la completa disponibilidad y no hay descuento en relación con el dinero en efectivo.

\section{REFERENCIAS BIBLIOGRÁFICAS}

Bagus, PH. (2009): «The Quality of Money.» Quarterly Journal of Austrian Economics, vol. 12, n. 4, pp. 41-64.

Bagus, PH. y Schiml, M.H. (2009): «New Modes of Monetary Policy: Qualitative Easing by the Fed.» Economic Affairs, vol. 29 , n. 2, pp. 46-49. 
Carver, T.N. (1934): «The Demand for Money.» The Economic Journal, vol. 44 (174), pp. 188-206.

Hazlitt, H. (1978): The Inflation Crisis, and How to Resolve It. New Rochelle, N.Y.: Arlington House.

Huerta De Soto, J. (1998): Dinero, crédito bancario y ciclos económicos. Madrid: Unión Editorial.

Menger, C. (1892): «On the Origin of Money.» Economic Journal, vol. 2, pp. 239-55.

- (1871): Grundsätze der Volkswirtschaftslehre. Vienna: Wilhelm Braumüller.

Mises, L. von (1912): Theorie des Geldes und der Umlaufsmittel. Leipzig: Duncker und Humblodt.

Rothbard, M.N. (2008): What has Government Done to Our Money? 5..$^{\mathrm{a}}$ ed. Auburn, Ala.: Ludwig von Mises Institute.

SÁnchez Martínez, L.C. (2009): «El mejor dinero posible», Procesos de Mercado: Revista Europea de Economía Política, vol. 6, n. 2, pp. 173-201.

TAgHiZADEGAN, R. (2008): Kritik der Freiwirtschaftslehre nach Silvio Gesell. Viena: Institut für Wertewirtschaft. http://www. wertewirtschaft.org/analysen/Freiwirtschaft.pdf

VARIAN, H.R. (2004): "Why is that Dollar Bill in Your Pocket Worth Anything?» New York Times. Enero 15. http:// people.ischool.berkeley.edu/ hal/people/hal/NYTimes/2 004-01-15.html. 